\title{
Parabolic Partial Differential Equations as Inverse Moments Problem
}

\author{
Maria B. Pintarelli1,2 \\ ${ }^{1}$ Grupo de Aplicaciones Matematicas y Estadisticas de la Facultad de Ingenieria (GAMEFI), Universidad Nacional \\ de La Plata (UNLP), La Plata, Argentina \\ ${ }^{2}$ Departamento de Matematica, Facultad de Ciencias Exactas, Universidad Nacional de La Plata (UNLP), La Plata, \\ Argentina \\ Email: mariabpintarelli@gmail.com
}

Received 10 December 2015; accepted 22 January 2016; published 25 January 2016

Copyright ( 2016 by author and Scientific Research Publishing Inc.

This work is licensed under the Creative Commons Attribution International License (CC BY).

http://creativecommons.org/licenses/by/4.0/

(c) () Open Access

\section{Abstract}

We considerer parabolic partial differential equations

under the conditions

$$
w_{t}-\left(w_{x}\right)_{x}=r(x, t)
$$

$$
\begin{aligned}
& w_{x}\left(a_{1}, t\right)=k_{1}(t) \quad w_{x}\left(b_{1}, t\right)=k_{2}(t) \\
& w\left(x, a_{2}\right)=h_{1}(t) \quad w\left(x, b_{2}\right)=h_{2}(t)
\end{aligned}
$$

on a region $E=\left(a_{1}, b_{1}\right) \times\left(a_{2}, b_{2}\right)$. We will see that we can write the equation in partial derivatives as an Fredholm integral equation of first kind and will solve this latter with the techniques of inverse moments problem. We will find an approximated solution and bounds for the error of the estimated solution using the techniques on moments problem. Also we consider the onedimensional one-phase inverse Stefan problem.

\section{Keywords}

Parabolic PDEs, Freholm Integral Equations, Generalized Moment Problem

\section{Introduction}

We considerer parabolic partial differential equation of the form:

$$
w_{t}-\left(w_{x}\right)_{x}=r(x, t)
$$


where the unknown function $w(x, t)$ is defined in $E=\left(a_{1}, b_{1}\right) \times\left(a_{2}, b_{2}\right) . r(x, t)$ is known function. We consider conditions

$$
\begin{aligned}
& w_{x}\left(a_{1}, t\right)=k_{1}(t) \quad w_{x}\left(b_{1}, t\right)=k_{2}(t) \\
& w\left(x, a_{2}\right)=h_{1}(t) \quad w\left(x, b_{2}\right)=h_{2}(t) .
\end{aligned}
$$

This problem was studied under conditions of Cauchy in [1].

Parabolic differential equations are commonly used in the fields of engineering and science for simulating physical processes. These equations describe various processes in viscous fluid flow, filtration of liquids, gas dynamics, heat conduction, elasticity, biological species, chemical reactions, environmental pollution, etc. [2] [3].

In a variety of cases, approximations are used to convert parabolic PDEs to ordinary differential equations or even to algebraic equations. The existence and uniqueness properties of this problem are presented in literature. Several numerical methods have been proposed for the solution of this problem [4]-[6].

Next section is devoted to showing how the differential equation (1) is transformed into integral equation of first kind that can be seen as generalized moments problem. In Section 3 there we present a theorem that guarantees under certain conditions the stability and convergence of the finite generalized moment problem. In Section 4 we exemplify the general method by applying it to some parabolic PDEs of the form (1). Finally in Section 5 the method is applied to solve the one-dimensional one-phase inverse Stefan problem.

The Stefan problem consists of finding $w$ y $s$ such that

$$
\begin{aligned}
& \frac{\partial w}{\partial t}=\frac{\partial^{2} w}{\partial x^{2}} \quad \text { in }\{(x, t) ; 0<x<s(t) ; t>0\} \\
& -\frac{\partial w}{\partial x}(0, t)=f(t) \quad t>0 \\
& w(s(t), t)=0 \quad t>0 \\
& \frac{\mathrm{d} s}{\mathrm{~d} t}=-\frac{\partial w}{\partial x}(s(t), t) \quad t>0 \\
& w(x, 0)=w_{0}(x) \quad x \geq 0 \\
& s(0)=a .
\end{aligned}
$$

The classical Stefan problem is a nonlinear initial value problem with a moving boundary whose position is unknown a priori and it must be determined as part of the solution. The differential equations of parabolic type governing heat diffusion with phase change are an important class of Stefan problems.

The direct Stefan problem requires determining both the temperature and the moving boundary interface when the initial and boundary conditions, and the thermal properties of the heat conducting body are known. Conversely, inverse Stefan problems require determining the initial and/or boundary conditions, and/or thermal properties from additional information which may involve the partial knowledge or measurement of the moving boundary interface position, its velocity in a normal direction, or the temperature at selected interior thermocouples of the domain.

In this paper we solve the inverse Stefan problem: find $f(t)$ with $s(t)$ known such that the above conditions are met.

The d-dimensional generalized moment problem [7]-[10] can be posed as follows: find a function $u$ on a domain $\Omega \subset \mathbf{R}^{d}$ satisfying the sequence of equations

$$
\int_{\Omega} u(x) g_{n}(x) \mathrm{d} x=\mu_{n} \quad n \in \mathbf{N}
$$

where $\left(g_{n}\right)$ is a given sequence of functions lying in $\mathbf{L}^{2}(\Omega)$ linearly independent.

Many inverse problems can be formulated as an integral equation of the first kind, namely,

$$
\int_{a}^{b} K(x, y) u(y) \mathrm{d} y=f(x) \quad x \in(a, b) .
$$


$K(x, y)$ and $f(x)$ are given functions and $u(y)$ is a solution to be determined; $f(x)$ is a result of experimental measurements and hence is given only at finite set of points. It follows that the above integral equation is equivalent to the following moment problem

$$
\int_{a}^{b} K\left(x_{n}, y\right) u(y) \mathrm{d} y=f\left(x_{n}\right) \quad n=1,2, \cdots .
$$

Also we considerer the multidimensional moment problems

$$
\int_{\Omega} K\left(x_{n}, y\right) u(y) \mathrm{d} y=f\left(x_{n}\right) \quad n=1,2, \cdots, \Omega \subset \mathbf{R}^{d} .
$$

Moment problems are usually ill-posed. There are various methods of constructing regularized solutions, that is, a approximate solution stable with respect to the given data. One of them is the method of truncated expansion.

The method of truncated expansion consists in approximating (2) by finite moment problems

$$
\int_{\Omega} u(x) g_{i}(x) \mathrm{d} x=\mu_{i} \quad i=1,2, \cdots, n .
$$

Solved in the subspace $\left\langle g_{1}, g_{2}, \cdots, g_{n}\right\rangle$ generated by $g_{1}, g_{2}, \cdots, g_{n}$ (3) is stable. Considering the case where the data $\mu=\left(\mu_{1}, \mu_{2}, \cdots, \mu_{n}\right)$ are inexact, we apply some convergence theorems and error estimates for the regularized solutions [9] [11].

\section{Parabolic Partial Differential Equations as Integral Equations of First Kind}

Let $F(w(x, t))=r(x, t)$ be a partial differential equations such as (1). The solution $w(x, t)$ is defined on the region $E=\left(a_{1}, b_{1}\right) \times\left(a_{2}, b_{2}\right)$ and verifies conditiones on the boundary $C=\partial E$ :

$$
\begin{aligned}
& w_{x}\left(a_{1}, t\right)=k_{1}(t) \quad w_{x}\left(b_{1}, t\right)=k_{2}(t) \\
& w\left(x, a_{2}\right)=h_{1}(t) \quad w\left(x, b_{2}\right)=h_{2}(t) .
\end{aligned}
$$

We apply the technique used in [1]. Let $F^{*}=\left(F_{1}(w), F_{2}(w)\right)$ be a vectorial field such that $w$ verifies $\operatorname{div}\left(F^{*}\right)=h^{*}(w)$ with $h^{*}$ a known function and, reciprocally, if $w$ verifies $\operatorname{div}\left(F^{*}\right)=h^{*}(w)$ then $F(w(x, t))=r(x, t)$.

Specifically in this case $F(w(x, t))=w_{t}-\left(w_{x}\right)_{x}$ and we take

$$
\begin{aligned}
& F^{*}=\left(F_{1}(w), F_{2}(w)\right)=\left(w_{x},-w\right) \\
& h^{*}(w)=-r(x, t) .
\end{aligned}
$$

Let $u(x, t, \tau, \xi)$ be the auxiliary function

$$
u(x, t, \tau, \xi)=\mathrm{e}^{-t \xi} \cos \left(\frac{x \pi}{b_{1}} \tau\right) .
$$

Since

$$
\operatorname{udiv}\left(F^{*}\right)=u h^{*}(w)
$$

we have

$$
\iint_{E} u \operatorname{div}\left(F^{*}\right) \mathrm{d} A=\iint_{E} u h^{*}(w) \mathrm{d} A
$$

Moreover, as

$$
u \operatorname{div}\left(F^{*}\right)=\operatorname{div}\left(u F^{*}\right)-F^{*} \cdot \nabla u
$$

and

$$
\iint_{E} u \operatorname{div}\left(F^{*}\right) \mathrm{d} A=\underbrace{\iint_{E} \operatorname{div}\left(u F^{*}\right) \mathrm{d} A}_{=\int_{C}\left(u F^{*}\right) \cdot n \mathrm{ds}}-\iint_{E} F^{*} \cdot \nabla u \mathrm{~d} A
$$


we obtain

$$
\iint_{E} u h^{*}(w) \mathrm{d} A=\int_{C}\left(u F^{*}\right) \cdot n \mathrm{~d} s-\iint_{E} F^{*} \cdot \nabla u \mathrm{~d} A
$$

where $\nabla u=\left(u_{\tau}, u_{\xi}\right)$.

We consider the integral

$$
\iint_{E} F^{*} \cdot \nabla u \mathrm{~d} A=\iint_{E} F_{1} u_{\tau}+F_{2} u_{\xi} \mathrm{d} A
$$

Integrating by parts:

$$
\begin{aligned}
\iint_{E} F_{1} u_{\tau} \mathrm{d} A=\int_{a_{2}}^{b_{2}} \int_{a_{1}}^{b_{1}} F_{1} u_{\tau} \mathrm{d} \tau \mathrm{d} \xi= & \int_{a_{2}}^{b_{2}}\left(w\left(b_{1}, \xi\right) u_{\tau}\left(x, t, b_{1}, \xi\right)-w\left(a_{1}, \xi\right) u_{\tau}\left(x, t, a_{1}, \xi\right)\right) \mathrm{d} \xi \\
& -\int_{a_{2}}^{b_{2}} \int_{a_{1}}^{b_{1}} w(\tau, \xi) u_{\tau \tau} \mathrm{d} \tau \mathrm{d} \xi .
\end{aligned}
$$

Note that in (9) if $x$ is a natural number then

$$
u_{\tau}\left(x, t, b_{1}, \xi\right)=-\mathrm{e}^{-t \xi} \frac{x \pi}{b_{1}} \sin \left(\frac{x \pi}{b_{1}} b_{1}\right)=0
$$

and if $a_{1}=0$ then

$$
u_{\tau}\left(x, t, a_{1}, \xi\right)=-\mathrm{e}^{-t \xi} \frac{x \pi}{b_{1}} \sin \left(\frac{x \pi}{b_{1}} a_{1}\right)=0
$$

Thus if $x \in N$ and $a_{1}=0$ :

$$
\iint_{E} F^{*} \cdot \nabla u d A=-\iint_{E}\left(w u_{\tau \tau}-w u_{\xi}\right) \mathrm{d} A=-\int_{a_{2}}^{b_{2}} \int_{a_{1}}^{b_{1}} w u\left(\left(\frac{x \pi}{b_{1}}\right)^{2}+t\right) \mathrm{d} \tau \mathrm{d} \xi .
$$

Also if we write $C=\partial E=C_{1} \cup C_{2} \cup C_{3} \cup C_{4}$ (see Figure 1) then

$$
\begin{aligned}
& \int_{C}\left(u F^{*}\right) \cdot n \mathrm{~d} s=\int_{C_{1}}\left(u F^{*}\right) \cdot n \mathrm{~d} s+\int_{C_{2}}\left(u F^{*}\right) \cdot n \mathrm{~d} s+\int_{C_{3}}\left(u F^{*}\right) \cdot n \mathrm{~d} s+\int_{C_{4}}\left(u F^{*}\right) \cdot n \mathrm{~d} s \\
& \int_{C_{1}}\left(u F^{*}\right) \cdot n \mathrm{~d} s=\int_{a_{1}}^{b_{1}} u\left(x, t, \tau, a_{2}\right) \underbrace{w\left(\tau, a_{2}\right)}_{h_{1}(\tau)} \mathrm{d} \tau \\
& \int_{C_{3}}\left(u F^{*}\right) \cdot n \mathrm{~d} s=-\int_{a_{1}}^{b_{1}} u\left(x, t, \tau, b_{2}\right) \underbrace{w\left(\tau, b_{2}\right)}_{h_{2}(\tau)} \mathrm{d} \tau \\
& \int_{C_{2}}\left(u F^{*}\right) \cdot n \mathrm{~d} s=\int_{a_{2}}^{b_{2}} u\left(x, t, b_{1}, \xi\right) \underbrace{w_{\tau}\left(b_{1}, \xi\right)}_{k_{2}(\xi)} \mathrm{d} \xi \\
& \int_{C_{4}}\left(u F^{*}\right) \cdot n \mathrm{~d} s=-\int_{a_{2}}^{b_{2}} u\left(x, t, a_{1}, \xi\right) \underbrace{\xi w_{\tau}\left(a_{1}, \xi\right)}_{k_{1}(\xi)} \mathrm{d} \xi . \\
& b_{2}
\end{aligned}
$$

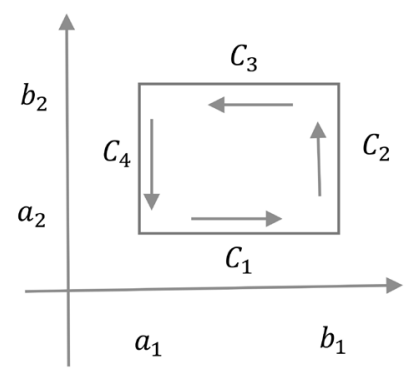

Figure 1. Domain $E$ and contour $\partial E=C$. 
We write

$$
\begin{gathered}
G(x, t)=\int_{C_{1}}\left(u F^{*}\right) \cdot n \mathrm{~d} s+\int_{C_{2}}\left(u F^{*}\right) \cdot n \mathrm{~d} s+\int_{C_{3}}\left(u F^{*}\right) \cdot n \mathrm{~d} s+\int_{C_{4}}\left(u F^{*}\right) \cdot n \mathrm{~d} s \\
R(x, t)=\iint_{E} r(\tau, \xi) u(x, t, \tau, \xi) \mathrm{d} A
\end{gathered}
$$

finally, if $x \in N$ y $a_{1}=0$ we get:

$$
\int_{a_{2}}^{b_{2}} \int_{a_{1}}^{b_{1}} w(\tau, \xi) u(x, t, \tau, \xi) \mathrm{d} \tau \mathrm{d} \xi=\frac{G(x, t)-R(x, t)}{\left(\left(\frac{x \pi}{b_{1}}\right)^{2}+t\right)}=\varphi(x, t) .
$$

If $E=\left\{(\tau, \xi) / \tau \geq 0 ; a_{2} \leq \xi \leq b_{2}\right\}$ then you can take

$$
u(x, t, \tau, \xi)=\mathrm{e}^{-t \xi} \cos (x \tau)
$$

and we must have $w(\tau, \xi) \rightarrow 0$ when $\tau \rightarrow \infty$.

\section{Solution of Generalized Moment Problems}

Equation (13) is of the form:

$$
\int_{a_{1}}^{b_{1}} \int_{a_{2}}^{b_{2}} w(\tau, \xi) K(x, t, \tau, \xi) \mathrm{d} \tau \mathrm{d} \xi=\varphi(x, t) .
$$

We assign natural values to $x$ and $t: x=i, i=0,1, \cdots, m$ and $t=j, j=0,1, \cdots, n$ and we consider the corresponding generalized finite moment problem bi-dimensional [12] [13]

$$
\int_{a_{1}}^{b_{1}} \int_{a_{2}}^{b_{2}} w(\tau, \xi) K_{i j}(\tau, \xi) \mathrm{d} \tau \mathrm{d} \xi=\varphi(i, j)=\mu_{i j} \quad i=0,1, \cdots, m \quad j=0,1, \cdots, n .
$$

To obtain a numerical approximation of the solution $w(\tau, \xi)$ the truncated expansion method is applied [9] [11].

We considerer the basis $\left\{\phi_{i}(\tau, \xi)\right\}_{i=0}^{\infty}$ obtained from the sequence $\left\{K_{i j}(\tau, \xi)\right\}$ with $i=0,1, \cdots, m$; $j=0,1, \cdots, n$ by Gram-Schmidt method and addition of the necessary functions in order to have an orthonormal basis.

To facilitate the calculations we write $\left\{K_{i}(\tau, \xi)\right\}_{i=0}^{r}$ and $\left\{\mu_{i}\right\}_{i=0}^{r}$ with $r=m \cdot n$.

We then approximate the solution $w(\tau, \xi)$ with

$$
p_{r}(\tau, \xi)=\sum_{i=0}^{r} \lambda_{i} \phi_{i}(\tau, \xi)
$$

and

$$
\lambda_{i}=\sum_{j=0}^{i} C_{i j} \mu_{j} \quad i=0,1, \cdots, r
$$

where the coefficients $C_{i j}$ verifies

$$
\begin{gathered}
C_{i j}=\left(\sum_{k=j}^{i-1}(-1) \frac{\left\langle K_{i}(\tau, \xi) \mid \phi_{k}(\tau, \xi)\right\rangle}{\left\|\phi_{k}(\tau, \xi)\right\|^{2}} C_{k j}\right) \cdot\left\|\phi_{i}(\tau, \xi)\right\|^{-1} \quad 1<i \leq r ; 1 \leq j<i \\
C_{i i}=\left\|\phi_{i}(\tau, \xi)\right\|^{-1} \quad i=0,1, \cdots, r .
\end{gathered}
$$

The proof of the following theorem is in [14].

Theorem 1. Let $\left\{\mu_{m}\right\}_{m=0}^{r}$ be a set of real numbers and let $\varepsilon$ and $E$ be two positive numbers such that

$$
\begin{aligned}
& \sum_{m=0}^{r}\left|\int_{a_{2}}^{b_{2}} \int_{a_{1}}^{b_{1}} K_{m}(\tau, \xi) w(\tau, \xi) \mathrm{d} \tau \mathrm{d} \xi-\mu_{m}\right|^{2} \leq \varepsilon^{2} \\
& \int_{a_{2}}^{b_{2}} \int_{a_{1}}^{b_{1}}\left[\left(b_{1}-a_{1}\right)^{2} w_{\tau}^{2}+\left(b_{2}-a_{2}\right)^{2} w_{\xi}^{2}\right] \mathrm{d} \tau \mathrm{d} \xi \leq E^{2}
\end{aligned}
$$


then

$$
\int_{a_{2}}^{b_{2}} \int_{a_{1}}^{b_{1}}|w(\tau, \xi)|^{2} \mathrm{~d} \tau \mathrm{d} \xi \leq \min _{m}\left\{\left\|C C^{\mathrm{T}}\right\| \varepsilon^{2}+\frac{E^{2}}{8(m+1)^{2}} ; m=0,1, \cdots, r\right\}
$$

where $C$ is the triangular matriz with elements $C_{i j} \quad(1<i \leq r ; 1 \leq j<i)$. And

$$
\int_{a_{2}}^{b_{2}} \int_{a_{1}}^{b_{1}}\left|p_{r}(\tau, \xi)-w(\tau, \xi)\right|^{2} \mathrm{~d} \tau \mathrm{d} \xi \leq\left\|C C^{\mathrm{T}}\right\| \varepsilon^{2}+\frac{E^{2}}{8(r+1)^{2}} .
$$

If $E=\left(a_{1}, \infty\right) \times\left(a_{2}, \infty\right)$, then (18) it is replaced by

and we must have

$$
\int_{a_{2}}^{\infty} \int_{a_{1}}^{\infty}\left[\tau w_{\tau}^{2}+\xi w_{\xi}^{2}\right] \operatorname{Exp}[\tau+\xi] \mathrm{d} \tau \mathrm{d} \xi \leq E^{2}
$$

and

$$
\tau^{m} w(\tau, \xi) \rightarrow 0 \quad \text { if } \tau \rightarrow \infty \quad \text { for all } m \in N
$$

$$
\xi^{n} w(\tau, \xi) \rightarrow 0 \quad \text { if } \xi \rightarrow \infty \quad \text { for all } n \in N
$$

\section{Numerical Examples}

\subsection{Example 1}

Let considerer the equation

$$
w_{t}-w_{x x}=0
$$

in the domain $E=(0,2) \times(0,2)$ and boundary condition on $\partial E$ given by

$$
\begin{gathered}
w_{x}(0, t)=\mathrm{e}^{-t} \quad w_{x}(2, t)=\cos (2) \mathrm{e}^{-t} \\
w(x, 0)=\sin (x) \quad w(x, 2)=\sin (x) \mathrm{e}^{-2} .
\end{gathered}
$$

The exact solution is $w(x, t)=\sin (x) \mathrm{e}^{-t}$.

In Figure 2 the approximate numerical solution and the exact one are compared.

Were taken $r=9$ moments.

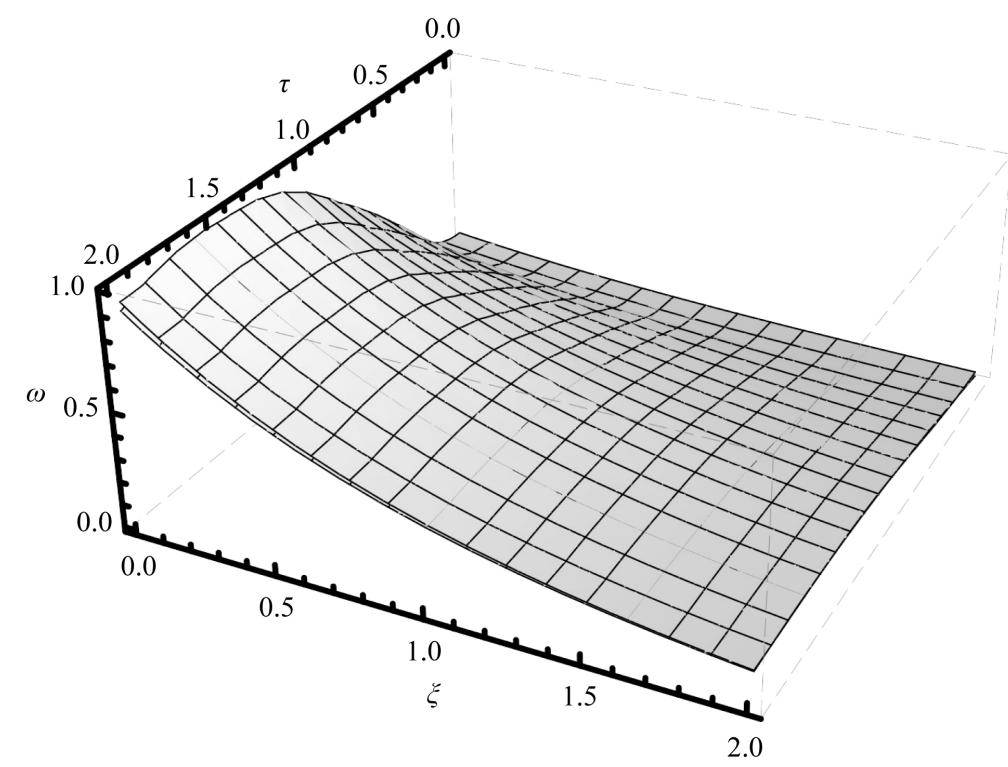

Figure 2. $w(x, t)=\sin (x) \mathrm{e}^{-t}$ and $p_{9}(x, t)$. 
The accuracy is, in this case $\int_{0}^{2} \int_{0}^{2}\left|p_{9}(x, t)-w(x, t)\right|^{2} \mathrm{~d} x \mathrm{~d} t=0.0361472$.

\subsection{Example 2}

Let considerer the equation

$$
w_{t}-w_{x x}=-4 \mathrm{e}^{-3 t+x}
$$

in the domain $E=(0,1) \times(0, \infty)$ and boundary condition on $\partial E$ given by

$$
\begin{gathered}
w_{x}(0, t)=\mathrm{e}^{-3 t} \quad w_{x}(1, t)=\mathrm{e}^{1-3 t} \\
w(x, 0)=\mathrm{e}^{x} .
\end{gathered}
$$

The exact solution is $w(x, t)=\mathrm{e}^{x-3 t}$.

In Figure 3 the approximate numerical solution and the exact one are compared. Were taken $r=9$ moments.

To apply Gram Schmidt to $\left\{K_{i j}(\tau, \xi)\right\}$ we consider the inner product

$$
\left\langle f_{i}(\tau, \xi), f_{j}(\tau, \xi)\right\rangle=\int_{a_{1}}^{b_{1}} \int_{a_{2}}^{\infty} f_{i}(\tau, \xi) f_{j}(\tau, \xi) \mathrm{e}^{-\xi} \mathrm{d} \xi \mathrm{d} \tau .
$$

The accuracy is, with this inner product $\left\|p_{9}(x, t)-w(x, t)\right\|^{2}=0.0250523$.

\section{The One-Dimensional One-Phase Inverse Stefan Problem}

\subsection{The Inverse One-Phase Stefan Problem as Integral Equation}

The Stefan problem consists of finding $w$ y s such that

$$
\begin{aligned}
& \frac{\partial w}{\partial t}=\frac{\partial^{2} w}{\partial x^{2}} \quad \text { in }\{(x, t) ; 0<x<s(t) ; t>0\} \\
& -\frac{\partial w}{\partial x}(0, t)=f(t) \quad t>0 \\
& w(s(t), t)=0 \quad t>0
\end{aligned}
$$

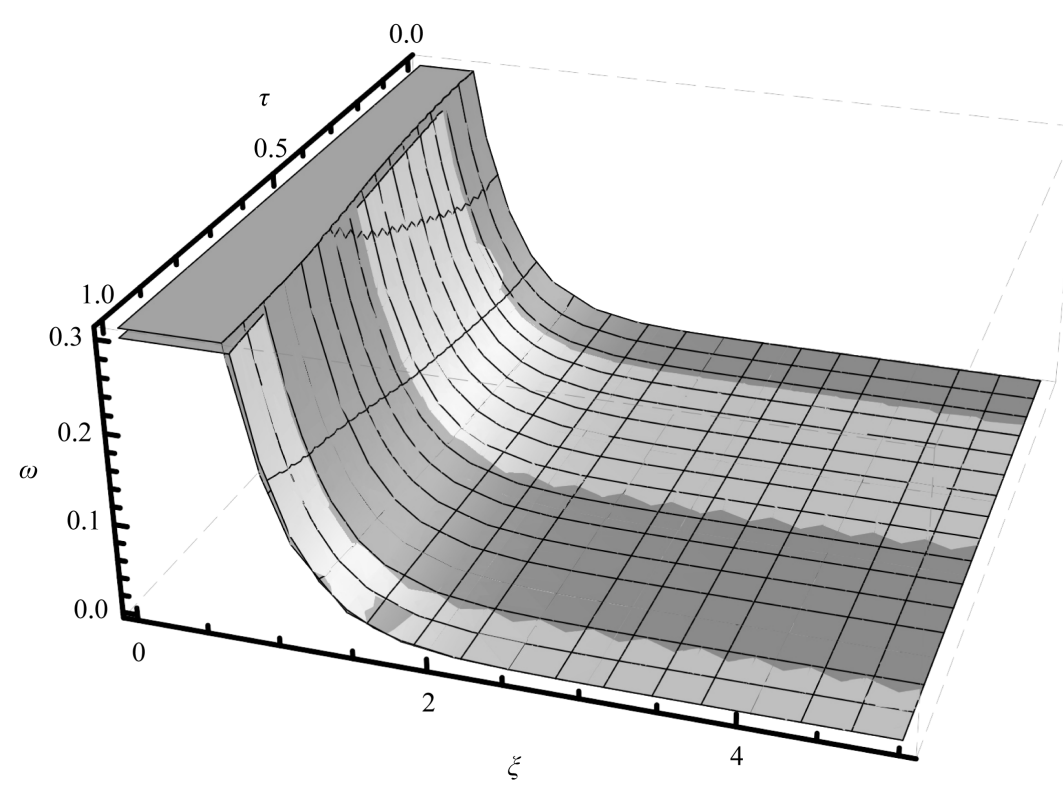

Figure 3. $w(x, t)=\mathrm{e}^{x-3 t}$ and $p_{9}(x, t)$. 


$$
\begin{aligned}
& \frac{\mathrm{d} s}{\mathrm{~d} t}=-\frac{\partial w}{\partial x}(s(t), t) \quad t>0 \\
& w(x, 0)=w_{0}(x) \quad x \geq 0 \\
& s(0)=a .
\end{aligned}
$$

We want to solve the inverse Stefan problem: to find $f(t)$ with $s(t)$ known such that the above conditions are met.

We write

$$
w_{\xi}-\left(w_{\tau}\right)_{\tau}=0 .
$$

We take the auxiliary function

$$
u(x, t, \tau, \xi)=\mathrm{e}^{-(t+1) \xi} \cos (x \tau) .
$$

Therefore

$$
\frac{\partial^{2} u}{\partial \tau^{2}}=u_{\tau \tau}=x^{2} u \quad \frac{\partial u}{\partial \xi}=u_{\xi}=-(t+1) u .
$$

We consider the vector field $F^{*}(w)=\left(F_{1}(w), F_{2}(w)\right)$ with $F_{1}(w)=w_{\tau}, F_{2}(w)=-w$. In this manner $\operatorname{div}\left(F^{*}(w)\right)=w_{\tau \tau}-w_{\xi}$. In consequence if $w$ it is solution of the Equation (27), then $\operatorname{div}\left(F^{*}(w)\right)=0$. Reciprocally, if $w$ satisfies $\operatorname{div}\left(F^{*}(w)\right)=0$, then $w$ it is solution of the Equation (27).

We write $E=\{(\tau, \xi) ; 0<\tau<s(\xi) ; \xi>0\}$. We use that

$$
\operatorname{div}\left(u F^{*}\right)=u \operatorname{div}\left(F^{*}\right)-F^{*} \cdot \nabla u
$$

if . it is the scalar product and $\nabla$ is the gradient operator we get

$$
\iint_{E} u \operatorname{div}\left(F^{*}\right) \mathrm{d} A=\iint_{E} \operatorname{div}\left(u F^{*}\right) \mathrm{d} A-\iint_{E} F^{*} \cdot \nabla u \mathrm{~d} A .
$$

By the divergence theorem

$$
\iint_{E} \operatorname{div}\left(u F^{*}\right) \mathrm{d} A=\int_{C}\left(u F^{*}\right) \cdot n \mathrm{~d} s
$$

with $C=\partial E$, in consequence

$$
\iint_{E} u \operatorname{div}\left(F^{*}\right) \mathrm{d} A=\int_{C}\left(u F^{*}\right) \cdot n \mathrm{~d} s-\iint_{E} F^{*} \cdot \nabla u \mathrm{~d} A .
$$

We calculate $\iint_{E} F^{*} \cdot \nabla u \mathrm{~d} A$ :

First we write

$$
F^{*} \cdot \nabla u=\left(F_{1}, F_{2}\right) \cdot\left(u_{\tau}, u_{\xi}\right)=F_{1} u_{\tau}+F_{2} u_{\xi}
$$

and $E_{T}=\{(\tau, \xi) ; 0<\tau<s(\xi) ; 0<\xi<T\}$, (Figure 4) then we take $T \rightarrow \infty$.

If $F_{1}^{p}(w)=\int F_{1}(w) \mathrm{d} \tau=w$ then

$$
\begin{aligned}
& \iint_{E_{T}} F_{1} u_{\tau} \mathrm{d} A=\int_{0}^{T} \int_{0}^{s(\xi)} F_{1} u_{\tau} \mathrm{d} \tau \mathrm{d} \xi \\
& =\int_{0}^{T}\left[F_{1}^{p}(w(s(\xi), \xi)) u_{\tau}(x, t, s(\xi), \xi)-F_{1}^{p}(0, \xi) u_{\tau}(x, t, 0, \xi)\right] \mathrm{d} \xi-\int_{0}^{T} \int_{0}^{s(\xi)} u_{\tau \tau} F_{1}^{p} \mathrm{~d} \tau \mathrm{d} \xi .
\end{aligned}
$$

As $w(s(\xi), \xi)=0$ by (23) and $u_{\tau}(x, t, 0, \xi)=x \sin (x \cdot 0) \mathrm{e}^{-(t+1) \xi}=0$ then

$$
\iint_{E_{T}} F^{*} \cdot \nabla u \mathrm{~d} A=-\int_{0}^{T} \int_{0}^{s(\xi)}\left(u_{\tau \tau} F_{1}^{p}(w)-F_{2}(w) u_{\xi}\right) \mathrm{d} \tau \mathrm{d} \xi=\int_{0}^{T} \int_{0}^{s(\xi)} u w\left(x^{2}+(t+1)\right) \mathrm{d} \tau \mathrm{d} \xi .
$$

Now we developed $\int_{C}\left(u F^{*}\right) \cdot n d s$. Observe the Figure 5 and: 


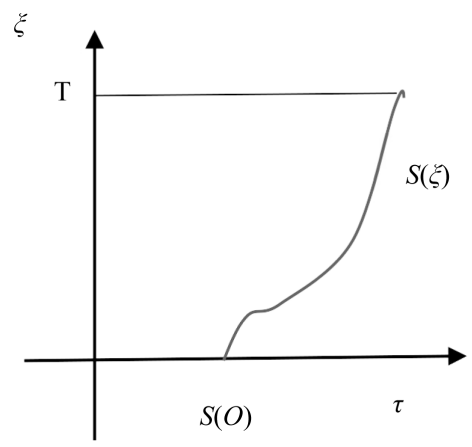

Figure 4. Domain $E_{T}$.

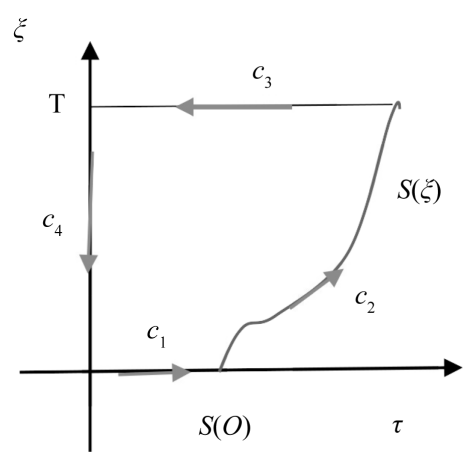

Figure 5. Domain $E_{T}$.

$$
\begin{aligned}
& \int_{c 1}\left(u F^{*}\right) \cdot n \mathrm{~d} s=\int_{0}^{s(0)} u(x, t, \tau, 0) w(\tau, 0) \mathrm{d} \tau=\int_{0}^{s(0)} u(x, t, \tau, 0) w_{0}(\tau) \mathrm{d} \tau \\
& \int_{c 3}\left(u F^{*}\right) \cdot n \mathrm{~d} s=\int_{0}^{s(T)} u(x, t, \tau, T) w(\tau, T) \mathrm{d} x \underset{T \rightarrow \infty}{\longrightarrow} 0 \\
& \int_{c 4}\left(u F^{*}\right) \cdot n \mathrm{~d} s=-\int_{0}^{T} u(x, t, 0, \xi) w_{\tau}(0, \xi) \mathrm{d} \xi \\
& \int_{c 2}\left(u F^{*}\right) \cdot n \mathrm{~d} s=-\int_{0}^{T} u(x, t, s(\xi), \xi) s_{\xi}(\xi) \mathrm{d} \xi
\end{aligned}
$$

Then

$$
\begin{aligned}
& \int_{0}^{\infty} \int_{0}^{s(\xi)} u w\left(x^{2}+(t+1)\right) \mathrm{d} \tau \mathrm{d} \xi \\
& =\int_{0}^{s(0)} \cos (x \tau) w(\tau, 0) \mathrm{d} \tau-\int_{0}^{\infty} \cos (x s(\xi)) \mathrm{e}^{-(t+1) \xi} s_{\xi}(\xi) \mathrm{d} \xi-\int_{0}^{\infty} \mathrm{e}^{-(t+1) \xi} w_{\tau}(0, \xi) \mathrm{d} \xi .
\end{aligned}
$$

To solve the inverse problem, where $s(\xi)$ is known and $w_{\tau}(0, \xi)$ is unknown we do $x^{2}+t+1=0$. In this manner:

$$
\begin{aligned}
& \int_{0}^{\infty} \mathrm{e}^{-(t+1) \xi} w_{\tau}(0, \xi) \mathrm{d} \xi \\
& =\int_{0}^{s(0)} \cos (\sqrt{-t-1} \tau) w(\tau, 0) \mathrm{d} \tau-\int_{0}^{\infty} \cos (\sqrt{-t-1} s(\xi)) \mathrm{e}^{-(t+1) \xi} s_{\xi}(\xi) \mathrm{d} \xi=\varphi(t) .
\end{aligned}
$$

We assign values to $t: t=0,1,2, \cdots$

$$
\int_{0}^{\infty} \mathrm{e}^{-(t+1) \xi} w_{\tau}(0, \xi) \mathrm{d} \xi=\varphi(t)=\mu_{t}
$$

We can interpret (35) as a one-dimensional generalized moments problem.

We solve the problem numerically considering the finite generalized moments problem 


$$
\int_{0}^{\infty} \mathrm{e}^{-(i+1) \xi} w_{\tau}(0, \xi) \mathrm{d} \xi=\varphi(i)=\mu_{i} \quad i=0,1, \cdots, n
$$

and we apply the truncated expansion method.

\subsection{Numerical Approximation to the Solution of the Inverse Stefan Problem}

To obtain a numerical approximation of the solution the procedure is analogous to that presented in Section 3. To approximate $w_{\tau}(0, \xi)$ is taken a base $\phi_{i}(\xi), i=0,1,2, \cdots, n$ of $L^{2}(0, \infty)$ obtained from the sequence $g_{i}(\xi)=\mathrm{e}^{-(i+1) \xi}$ by Gram-Schmidt method and necessary functions are added in order to have an orthonormal basis. We then approximate the solution $w_{\tau}(0, \xi)$ with:

$$
p_{n}(\xi)=\sum_{i=0}^{n} \lambda_{i} \phi_{i}(\xi)
$$

where

$$
\lambda_{i}=\sum_{j=0}^{i} C_{i j} \mu_{j} \quad i=0,1, \cdots, n
$$

and the coefficients $C_{i j}$ verifies

$$
\begin{gathered}
C_{i j}=\left(\sum_{r=j}^{i-1}(-1) \frac{\left\langle g_{i}(\xi) \mid \phi_{r}(\xi)\right\rangle}{\left\|\phi_{r}(\xi)\right\|^{2}} C_{r j}\right) \cdot\left\|\phi_{i}(\xi)\right\|^{-1} \quad 1<i \leq n ; 1 \leq j<i \\
C_{i i}=\left\|\phi_{i}(\xi)\right\|^{-1} \quad i=0,1, \cdots, n .
\end{gathered}
$$

The following theorem is the one-dimensional version of Theorem 1. In [15] is the demonstration when the domain is bounded.

We present here the demonstration when the domain is the interval $(0, \infty)$.

Theorem 2. Let $\left\{\mu_{i}\right\}_{i=0}^{n}$ be a set of real numbers and let $\varepsilon$ and $M$ be two positive numbers such that

$$
\begin{aligned}
& \sum_{i=0}^{n}\left|\int_{0}^{\infty} g_{i}(\xi) x(\xi) \mathrm{d} \xi-\mu_{i}\right|^{2} \leq \varepsilon^{2} \\
& \int_{0}^{\infty}\left[\xi \mathrm{e}^{\xi} x_{\xi}^{2}(\xi)\right] \mathrm{d} \xi \leq M^{2}
\end{aligned}
$$

then if $\lim _{\xi \rightarrow \infty} \xi^{r} x(\xi)=0, \forall r \in N$

$$
\int_{0}^{\infty}|x(\xi)|^{2} \mathrm{~d} \xi \leq \min _{n}\left\{\left\|C^{\mathrm{T}} C\right\| \varepsilon^{2}+\frac{M^{2}}{(i+1)} ; i=0,1, \cdots, n\right\}
$$

and

$$
\int_{0}^{\infty}\left|x(\xi)-p_{n}(\xi)\right|^{2} \mathrm{~d} \xi \leq\left\|C^{\mathrm{T}} C\right\| \varepsilon^{2}+\frac{M^{2}}{(n+1)}
$$

where $C$ is the triangular matriz with elements $C_{i j} \quad(1<i \leq n ; 1 \leq j<i)$.

Proof. Since the problem is linear we can assume $\mu_{i}=0, i=0,1, \cdots, n$.

We applied Gram-Scmidt method on $\left\{g_{i}(\xi)\right\}_{i=0}^{n}$ in $L^{2}(0, \infty)$ and we get $\left\{\phi_{i}(\xi)\right\}_{i=0}^{\infty}$ then add the resulting set of necessary functions to obtain an orthonormal basis.

We write $x(\xi)$ as

$$
x(\xi)=h_{n}(\xi)+f_{n}(\xi)
$$

where $h_{n}(\xi)$ it is the orthogonal projection of $x(\xi)$ on the linear space generated by the set $\left\{g_{i}(\xi)\right\}_{i=0}^{n}$ and $f_{n}(\xi)=x(\xi)-h_{n}(\xi)$ it is the orthogonal projection of $x(\xi)$ on the orthogonal complement. Here the underlying structure is the space $L^{2}(0, \infty)$. We can write 


$$
h_{n}(\xi)=\sum_{i=0}^{n} \lambda_{i} \phi_{i}(\xi) \quad f_{n}(\xi)=\sum_{i=n+1}^{\infty} \lambda_{i} \phi_{i}(\xi)
$$

where $\lambda_{i}=\int_{0}^{\infty} \phi_{i}(\xi) x(\xi) \mathrm{d} \xi, i=0,1, \cdots \quad$ are the Fourier coefficients in the expansion of $x(\xi)$.

To estimate $h_{n}(\xi)$ we consider the relationship between the Fourier coefficients $\lambda_{i}$ and the moments $\mu_{i}=\int_{0}^{\infty} g_{i}(\xi) x(\xi) \mathrm{d} t$ :

$$
\lambda_{i}=\sum_{j=0}^{i} C_{i j} \mu_{j} \quad i=0,1, \cdots, n
$$

where $C_{i j}$ they are given in (37) y (38).

In matrix notation

$$
\lambda=\left(\begin{array}{c}
\lambda_{0} \\
\lambda_{1} \\
\vdots \\
\lambda_{n}
\end{array}\right) \quad \mu=\left(\begin{array}{c}
\mu_{0} \\
\mu_{1} \\
\vdots \\
\mu_{n}
\end{array}\right) .
$$

Then

$$
\sum_{j=0}^{i} C_{i j} g_{j}(\xi)=\phi_{i}(\xi) \quad i=0,1, \cdots, n
$$

By (43) until ( 46) we can write

$$
\int_{0}^{\infty}\left|h_{n}(\xi)\right|^{2} \mathrm{~d} \xi=\langle\lambda \mid \lambda\rangle=\left\langle C^{\mathrm{T}} C \mu \mid \mu\right\rangle \leq\left\|C^{\mathrm{T}} C\right\|\|\mu\|^{2} \leq\left\|C^{\mathrm{T}} C\right\| \varepsilon^{2} .
$$

To estimate $f_{n}$ we see that each element of the orthonormal set $\phi_{i}(t)$ can be expanded in terms of the elements other orthonormal basis, in particular the base $\left\{l_{i}(\xi)=L_{i}(\xi) \mathrm{e}^{-\xi}\right\}_{i=0}^{\infty}$, where $L_{i}(\xi)$ it represents the Laguerre polynomial of degree $i$.

These polynomials satisfy

$$
\xi \frac{\mathrm{d}^{2}}{\mathrm{~d} \xi^{2}}\left[L_{i}(\xi)\right]+(1-\xi) \frac{\mathrm{d}}{\mathrm{d} \xi}\left[L_{i}(\xi)\right]+i L_{i}(\xi)=0
$$

or also

$$
-\frac{\mathrm{d}}{\mathrm{d} \xi}\left[\xi \frac{\mathrm{d}}{\mathrm{d} \xi}\left[L_{i}(\xi)\right]\right]+\xi \frac{\mathrm{d}}{\mathrm{d} \xi}\left[L_{i}(\xi)\right]=i L_{i}(\xi) .
$$

Then

$$
\phi_{i}(\xi)=\sum_{j=0}^{\infty} \gamma_{i j} l_{j}(\xi) \quad i=0,1,2, \cdots, n
$$

then using (50)

$$
f_{n}(\xi)=\sum_{i=n+1}^{\infty} \lambda_{i} \phi_{i}(\xi)=\sum_{i=n+1}^{\infty} \sum_{j=0}^{\infty} \lambda_{i} \gamma_{i j} l_{j}(\xi) .
$$

After several calculations

$$
\int_{0}^{\infty}\left|f_{n}(\xi)\right|^{2} \mathrm{~d} \xi=\left\|f_{n}(\xi)\right\|^{2}=\sum_{i=n+1}^{\infty}\left|\sum_{j=0}^{\infty} \lambda_{j} \gamma_{j i}\right|^{2}
$$

and 


$$
\int_{0}^{\infty}\left|f_{n}(\xi)\right|^{2} \mathrm{~d} \xi \leq \frac{1}{n+1} \sum_{i=0}^{\infty}(i+1)|\chi(i)|^{2}
$$

where

$$
\chi(i)=\sum_{j=0}^{\infty} \lambda_{j} \gamma_{j i}
$$

Now multiplying by $x(\xi)$ and integrating both sides of the differential Equation (49) and assuming that $\lim _{\xi \rightarrow \infty} \xi^{r} x(\xi)=0, \forall r \in N$ we get:

$$
\int_{0}^{\infty} \xi x_{\xi}^{2}(\xi) \mathrm{e}^{\xi} \mathrm{d} \xi=\sum_{i=0}^{\infty}(i+1) \chi^{2}(i)
$$

then by (53) and (55):

$$
\int_{0}^{\infty}\left|f_{n}(\xi)\right|^{2} \mathrm{~d} \xi \leq \frac{1}{n+1} \int_{0}^{\infty} \xi x_{\xi}^{2}(\xi) \mathrm{e}^{\xi} \mathrm{d} \xi \leq \frac{M^{2}}{n+1}
$$

from (47) and (56):

$$
\int_{0}^{\infty}|x(\xi)|^{2} \mathrm{~d} \xi \leq\left\|C^{\mathrm{T}} C\right\| \varepsilon^{2}+\frac{M^{2}}{(n+1)} .
$$

This inequality remains valid if we replace any integer $i$ between 0 and $n$ for $n$. Then the result (41) it demonstrated. An analogous demonstration proves inequality (42).

\section{Numerical Example}

Find $w_{\tau}(0, \xi)$ such that

$$
\begin{aligned}
& \frac{\partial w}{\partial \xi}=\frac{\partial^{2} w}{\partial \tau^{2}} \text { in }\{(\tau, \xi) ; 0<\tau<s(\xi) ; \xi>0\} \quad \text { with } s(\xi)=\sqrt{1+\xi} \\
& w(s(\xi), \xi)=0 \quad \xi>0 \\
& \frac{\mathrm{d} s}{\mathrm{~d} \xi}=-\frac{\partial w}{\partial \tau}(s(\xi), \xi) \quad \xi>0 \\
& w(\tau, 0)=w_{0}(\tau)=0.5 \mathrm{e}^{0.25} \sqrt{\pi}\left[\operatorname{erf}(0.25)-\operatorname{erf}\left(\frac{x}{2}\right)\right] \quad \tau \geq 0 .
\end{aligned}
$$

The exact solution is $w_{\tau}(0, \xi)=-\frac{\mathrm{e}^{0.25}}{2} \sqrt{\frac{1}{1+\xi}}$.

In Figure 6 the approximate numerical solution and the exact one are compared.

Were taken $r=6$ moments.

To apply Gram Schmidt to $\left\{g_{i}(\xi)\right\}_{i=0}^{n}$ en $L^{2}(0, \infty)$ we considerer the inner product

$$
\left\langle f_{i}(\xi), f_{j}(\xi)\right\rangle=\int_{0}^{\infty} f_{i}(\xi) f_{j}(\xi) \mathrm{e}^{-\xi} \mathrm{d} \xi .
$$

The accuracy is, with this inner product $\left\|p_{6}(\xi)-w_{\tau}(0, \xi)\right\|^{2}=0.00306424$.

\section{Conclusions}

The parabolic partial differential equations

$$
w_{\xi}-\left(w_{\tau}\right)_{\tau}=r(\tau, \xi)
$$

on a region $E=\left(0, b_{1}\right) \times\left(a_{2}, b_{2}\right)$ can be written as an Fredholm integral equation 


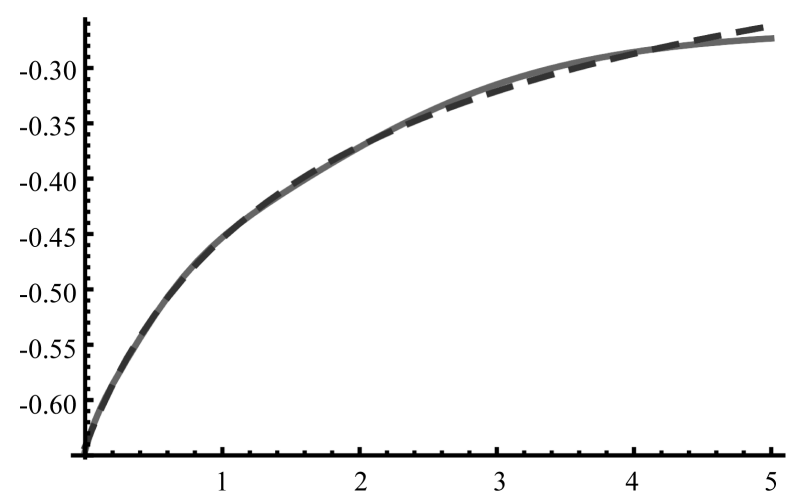

Figure 6. $w_{\tau}(0, \xi)=-\frac{\mathrm{e}^{0.25}}{2} \sqrt{\frac{1}{1+\xi}}$ and $p_{6}(\tau, \xi)$.

$$
\int_{a_{2}}^{b_{2}} \int_{0}^{b_{1}} w(\tau, \xi) u(x, t, \tau, \xi) \mathrm{d} \tau \mathrm{d} \xi=\frac{G(x, t)-R(x, t)}{\left(\left(\frac{x \pi}{b_{1}}\right)^{2}+t\right)}=\varphi(x, t) \quad x \in N .
$$

This equation is of the form:

$$
\int_{0}^{b_{1}} \int_{a_{2}}^{b_{2}} w(\tau, \xi) K(x, t, \tau, \xi) \mathrm{d} \tau \mathrm{d} \xi=\varphi(x, t)
$$

with $K(x, t, \tau, \xi)=\mathrm{e}^{-t \xi} \cos \left(\frac{x \pi}{b_{1}} \tau\right), x \in N$.

If $w(\tau, \xi) \in L^{2}(E)$, then this Fredholm integral equation of first kind can be transformed into a bi-dimensional generalized moment problem assigning integer values greater than or equal to zero to variables $x$ and $t$

$$
\int_{0}^{b_{1}} \int_{a_{2}}^{b_{2}} w(\tau, \xi) K_{i j}(\tau, \xi) \mathrm{d} \tau \mathrm{d} \xi=\mu_{i j} \quad i=0,1,2, \cdots, j=0,1,2, \cdots
$$

As the functions $\left\{K_{i j}(\tau, \xi)\right\}_{i j}$ are linearly independent then the generalized moment problem defined by (58) can be solved numerically considering the correspondent finite problem.

The inverse Stefan problem which it is to find $w_{\tau}(0, \xi)$ being $w(\tau, \xi)$ unknown and such that the following conditions are met

$$
\begin{aligned}
& \frac{\partial w}{\partial \xi}=\frac{\partial^{2} w}{\partial \tau^{2}} \quad \text { in }\{(\tau, \xi) ; 0<\tau<s(\xi) ; \xi>0\} \\
& -\frac{\partial w}{\partial \tau}(0, \xi)=f(\xi) \quad \xi>0 \\
& w(s(\xi), \xi)=0 \quad \xi>0 \\
& \frac{\mathrm{d} s}{\mathrm{~d} \xi}=-\frac{\partial w}{\partial \tau}(s(\xi), \xi) \quad \xi>0 \\
& w(\tau, 0)=w_{0}(\tau) \quad \tau \geq 0 \\
& s(0)=a
\end{aligned}
$$

is equivalent to solve the integral equation

$$
\begin{aligned}
& \int_{0}^{\infty} \mathrm{e}^{-(t+1) \xi} w_{\tau}(0, \xi) \mathrm{d} \xi \\
& =\int_{0}^{s(0)} \cos (\sqrt{-t-1} \tau) w(\tau, 0) \mathrm{d} \tau-\int_{0}^{\infty} \cos (\sqrt{-t-1} s(\xi)) \mathrm{e}^{-(t+1) \xi} s_{\xi}(\xi) \mathrm{d} \xi=\varphi(t)
\end{aligned}
$$


which is equivalent to the generalized moments problem

$$
\int_{0}^{\infty} \mathrm{e}^{-(i+1) \xi} w_{\tau}(0, \xi) \mathrm{d} \xi=\varphi(i)=\mu_{i} \quad i=0,1,2, \cdots
$$

and can be solved numerically considering the correspondent finite problem.

\section{References}

[1] Pintarelli, M.B. and Vericat, F. (2014) Partial Differential Equations as Three-Dimensional Inverse Problem of Moments. Journal of Mathematics and System Science, 4, 657-666.

[2] Cherniha, R.M. (2011) New Exact Solutions of One Nonlinear Equation in Mathematical Biology and Their Properties. Ukrainian Mathematical Journal, 53, 393-411.

[3] Mittal, R.C. and Jiwari, R. (2011) A Higher Order Numerical Scheme for Some Nonlinear Differential Equations: Models in Biology. International Journal for Computational Methods in Engineering Science and Mechanics, 12, 134140. http://dx.doi.org/10.1080/15502287.2011.564265

[4] Forsythe, G.E. and Wasow, W.R. (1960) Finite Difference Methods for Partial Differential Equations. John Wiley and Sons, New York.

[5] Zafarullah, A. (1971) Some Stable Implicit Difference Methods for Heat Equation with Derivative Boundary Condition. The Computer Journal, 14, 309-311. http://dx.doi.org/10.1093/comjnl/14.3.309

[6] Keast, P. and Mitchell, A.R. (1966) On the Instability of the Crank Nicholson Formula under Derivative Boundary Conditions. The Computer Journal, 9, 110-114. http://dx.doi.org/10.1093/comjnl/9.1.110

[7] Akheizer, N.I. (1965) The Classical Moment Problem. Olivier and Boyd, Edinburgh.

[8] Akheizer, N.I. and Krein, M.G. (1962) Some Questions in the Theory of Moment. American Mathematical Society, Providence.

[9] Ang, D.D., Gorenflo, R., Le, V.K. and Trong, D.D. (2002) Moment Theory and Some Inverse Problems in Potential Theory and Heat Conduction. Lectures Notes in Mathematics, Springer-Verlag, Berlin. http://dx.doi.org/10.1007/b84019

[10] Shohat, J.A. and Tamarkin, J.D. (1943) The Problem of Moments. Mathematics Survey, American Mathematical Society, Providence. http://dx.doi.org/10.1090/surv/001

[11] Talenti, G. (1987) Recovering a Function from a Finite Number of Moments. Inverse Problems, 3, 501-517. http://dx.doi.org/10.1088/0266-5611/3/3/016

[12] Pintarelli, M.B. and Vericat, F. (2011) Bi-Dimensional Inverse Moment Problems. Far East Journal of Mathematical Sciences, 54, 1-23.

[13] Pintarelli, M.B. and Vericat, F. (2012) Klein-Gordon Equation as a Bi-Dimensional Moment Problem. Far East Journal of Mathematical Sciences, 70, 201-225.

[14] Pintarelli, M.B. (2015) Linear Partial Differential Equations of First Order as Bi-Dimensional Inverse Moment Problem. Applied Mathematics, 6, 979-989. http://dx.doi.org/10.4236/am.2015.66090

[15] Pintarelli, M.B. and Vericat, F. (2008) Stability Theorem and Inversion Algorithm for a Generalized Moment Problem. Far East Journal of Mathematical Sciences, 30, 253-274. 